\title{
Feedback from the Eighth International Conference on AIDS, Amsterdam 1992
}

\begin{abstract}
Introduction
Following the Eighth International AIDS Conference in Amsterdam in July 1992 a feedback day for genitourinary medicine physicians was held at the Royal Zoological Society, London. We have followed the same convention as last year for citing abstracts, and used the numbers from the published abstract books. One of the contributors has cited references from the Fourth Neuroscience of HIV Infection and these are prefaced with the letters "NS".
\end{abstract}

\section{Epidemiology—Richard Keenlyside Worldwide spread}

Dr Jonathan Mann of the Harvard Aids Institute and Dr Michael Merson of the WHO Global Programme on AIDS reported that AIDS has now penetrated to almost all parts of the world and the spread of HIV has not been stopped in any community or country. In early 1992 at least 12.9 million persons worldwide were estimated to be infected with HIV $(7 \cdot 1$ million men and $4 \cdot 7$ million women). Of these about one in five has developed AIDS and 2.5 million have died. Cases in women have continued to increase dramatically from $25 \%$ of the total in 1990 to nearly $40 \%$ expected in 1992. By 1995 the number of children orphaned by AIDS may increase from 1.8 million to 3.7 million.

\section{South East Asia Oceania}

Within a few years the epidemic has spread through Thailand and has become established in India, Burma, China, the Philippines, Fiji and Samoa. The epidemic in Thailand and neighbouring countries has been closely studied. After an explosive increase in infection among incarcerated injecting drug users (3-5\% per month in 1987-8), it later spread to female sex workers (prevalence now 15\%) and their male clients (prevalence 5\%). A concurrent increase from $0.5 \%$ to $2.9 \%$ among young military recruits indicated rapid heterosexual spread which was linked in behavioural studies to early encounters with prostitutes and non use of condoms. (PoC4087, PoC4083, PoC4084)

The epidemic has evolved in a similar fashion in India but with rapid and earlier heterosexual spread. In Bombay, and other large cities, up to $60 \%$ of prostitutes, $10 \%$ of their clients and $38 \%$ of general sexually transmitted disease (STD) clinic attenders may be infected. Over three years, the prevalence among young male volunteer blood donors has increased from $0.04 \%$ to $0.5 \%$, but it is alarmingly high (86\%) among a group of homeless paid professional donors who also had high rates of hepatitis B (32\%) and syphilis $(21 \%)$ infection. Infection among drug users is a focal but growing problem. In a small area in north east India, on the Myanmar (formerly Burma) border, $76 \%$ of drug users (IDUs) and $6 \%$ of their female heterosexual contacts were found to be positive. (MoC93, ThC1554, PoC4070, PoC4087, PoC4171, PoC4623).

Latin America In a review of 60 studies of male and female sex workers in Latin America between 1987-90 transmission patterns were more complex (PoC4039). Here, women are commonly infected through contact with the bisexual partners of groups of highly infected male prostitutes. Transmission via IDUs has been moderate $(7 \%$ of reported cases) and blood transfusion related cases have declined, except in Mexico where paid donors have higher rates of infection. So far there has been limited growth in transmission from female prostitutes who have a low prevalence of infection (0-7\%) (PoC4065, PoC4036).

In the United States in mid 1992, there were at least 260000 persons living with AIDS and 40-80 000 new infections were expected last year. By 1994 there may be 415000 535000 cases and $320000-385000$ deaths reported and by then approximately one sixth of the cases will be in women. Cases in homosexual men appear to have levelled off in the north east but increased in the south. Heterosexually acquired cases continue to increase in all areas except the south and in 1989 AIDS was the sixth leading cause of death in women aged 25-44. The cost of AIDS in the US was 10.3 billion dollars in 1992 and is expected to reach 13.5 billion by 1994.

In Europe, 75000 cases of AIDS were reported by 1990 and heterosexually acquired infections are also increasing. Since 1987 more women than men have been reported in Sweden with heterosexually acquired infections (PoC4001), and in Switzerland unprotected heterosexual contact is a growing exposure factor for infection (PoC4004). In Milan, which has the highest AIDS incidence and mortality rates in Italy, $35 \%$ of the deaths in men aged 25-29 years in 1989 were from AIDS, overtaking overdose and road accidents as causes (PoC4633). Similarly, in Spain, deaths from AIDS in young people may soon become the principal cause of years of potential life lost (PuC3082).

In Africa the number of HIV cases has tripled in the past five years and has reached more than 7.5 million. Ongoing surveys in rural areas of Uganda have shown sustained high 
prevalences of infection in certain groups $(34 \%$ in young adults and $22 \%$ in women) but in the overall population the prevalence has remained fairly stable at 2-5\% over the past 3 years. (PoC4019, PoC4021)

\section{HIV-2 infection.}

Although HIV-2 was formerly confined to West African countries it has now been reported from Portugal, France, Mexico, North America, Argentina, and recently among STD clinic patients and prostitutes in Bombay, India (PoC4623 PoA2055). HIV-1 is also spreading into the region. In a report from Senegal HIV-1 was more common than HIV-2 in certain groups (ie hospital patients, those with tuberculosis (TB) and STD and prostitutes) in Dakar compared with rural areas (PoC1066). Studies of the natural history of HIV-1 and HIV-2 infections in Senegal and Cote D'Ivoire have shown that HIV-1 infections progress more rapidly to AIDS, CDC group IV disease and death than HIV-2 but the patterns of opportunistic infections and pathological findings at autopsy appear similar. In Abijan, the commonest causes of death at necropsy in 261 AIDS cases from both viruses, were: tuberculosis (34\%), cerebral toxoplasmosis (15\%) and bacteraemia (11\%); $74 \%$ died with wasting, and pneumocystis (2.0\%) and Kaposi's sarcoma (1.6\%) were uncommon (WeC1066, WeC1067 PoB3751).

\section{Tuberculosis and HIV}

The HIV pandemic has had a major impact on the epidemiology of TB worldwide and there were several reports of its resurgence in Africa and among urban populations in the US. In Central Africa, prospective case control studies have shown that $30-40 \%$ of TB cases are attributable to HIV infection and the risk of developing active TB is ten times greater in those with HIV infection. (PoC4023, PoC4033, PoC4417, TuC0566). Several reports stressed the need to screen patients with HIV for TB, and vice versa (TuC0571, PoC4665). In Nairobi the prevalence of HIV in TB patients increased from $7 \cdot 5 \%$ to $42 \%$ between $1985-90$ and the annual cost of treating TB cases almost doubled from $\$ 11,971$ in 1985 to $\$ 23,000$ in 1990. Relapses after treatment with recommended regimens occurred in up to $17 \%$ of cases and there was thiacetazone sensitivity leading to severe and sometimes fatal skin reactions in approximately $20 \%$ of patients. (PoB3090, PoB3086, PoB3859). There have been at least six large nosocomial outbreaks of multi-drug resistant TB reported in the USA; $82-94 \%$ of cases also had HIV infection and in $94 \%$ of cases the organism was resistant to isoniazid and rifampin. (TuB0535, TuC0568, TuB0534). HIV infection amplified the risk of progression to active disease and the case fatality rate was high (up to $89 \%$ died within $1-4$ months). In one outbreak, a cluster of 59 health care workers had positive tuberculin skin tests after exposure to a prisoner with TB and one developed active disease.

\section{Transmission in health care settings}

In the US, $4.8 \%$ of reported AIDS cases are in health care workers (HCW) but they are similar to non-HCW in terms of their exposure factors and most had non occupational risk factors. By May 1992, 29 documented seroconversions following accidental injury to HCW have been reported to the Centres for Disease Control; $76 \%$ were female and 3 had progressed to AIDS. The majority (83\%) were percutaneous exposures to blood and in $23(79 \%)$ cases there was an acute seroconversion illness. Another $63 \mathrm{HCW}$ with HIV infection and no other risk factors have also been reported (PoC4143).

Several surveys of HIV prevalence in selected occupational groups have shown very low levels (for example $0.06-0.09 \%$ of US physicians and surgeons and $0.03 \%$ of blood transfusion staff) and most health care workers found to be infected had non-occupational exposure factors. From pooled prospective studies worldwide the risk of a single exposure is estimated to be approximately $0.3 \%$. (PoC4131 PoC4130).

\section{Epidemiology II-Andrew N Phillips \\ Transmission of HIV}

The determinants of horizontal transmission of HIV continue to be studied extensively by epidemiologists. In the simplest kinds of study the prevalence of HIV infection is assessed in a sample and then the factors associated with being infected are evaluated. Several such studies were presented at the conference. The principal factors found to be associated with HIV infection among groups where transmission is principally heterosexual were the history of other sexually transmitted diseases (PoC4091, PoC4178, PoC4322, PoC4334, PoC4338, PoC4715, MoC0028, WeC1028, ThC1556, ThC1561) -ulcerative or otherwise-and, in males, lack of circumcision (PoC4091, PoC4161, PoC4299, PoC4346, ThC1514). In general, these factors persisted after adjustment for other factors considered. Even given their consistency, studies such as these leave doubts about whether these factors actually have a biological effect on transmission. A history of other STDs could just be acting as a measure of the level of exposure that an individual has had to HIV. Confounding could similarly produce the association between HIV status and lack of circumcision. Due to its association with certain religions, circumcised individuals in a community often differ from uncircumcised individuals in social and behavioural characteristics which could potentially influence HIV risk.

Heterosexual partner studies-where the HIV serostatus of partners of known infected people is evaluated-allow some further investigation of the question of the influence of other STDs. The fact that all subjects under consideration (that is, the contacts) are 
known to have been exposed to HIV helps to standardise for this confounding factor. Some previously reported partner studies ${ }^{1}$ and one presented at the conference found associations between infection with HIV and a history of other STDs (WeC1089); however, the other eight such studies presented in Amsterdam did not report such associations (PoC4152, PoC4165, PoC4155, PoC4156, PoC4164, PoC4355, PoC4530, PoC4561). Nonetheless, it is not clear from many of these latter studies whether the association between other STDs and transmission of HIV was assessed.

A finding from recent transmission studies was heavily supported by reports at the conference. More advanced infection in the index partner-as measured by the presence of symptoms, the CD4 lymphocyte count and p24 antigen status-showed a strong relationship with risk of transmission (PoC4152, PoC4165, PoC4155, PoC4164, PoC4530). Other studies found cigarette smoking (PoC4338), and oral contraceptive use (PoC4309, PoC4338, PoC4355) to be associated with increased risk of transmission. However, another study found a significant protective effect of oral contraceptive use (PoC4651).

On a virological level Simmonds and colleagues reported that, based on results from a partner study in Edinburgh, transmission of HIV is enhanced in the presence of strains of HIV containing certain sequences in the viral envelope gene. Sequence variation in this region, which lies in the V3 loop of the envelope, has been previously shown to be associated with cell tropism of the virus (WeC1086).

Transmission via injecting drug use was found to be raised in people who practised "backloading"-extracting drug from one syringe into another in New York drug users (ThC1551).

There was an interesting session on genetic variability in HIV within and between populations. One presentation came from Thailand. The V3 loop was sequenced from virus obtained from $\mathbf{5 0}$ individuals in seven provinces across the country (WeC1025). The isolates fell into two distinct groupstype $A$ was closer to African strains while type $B$ was more similar to strains seen in North America and Europe. Of those presumably infected sexually $91 \%(20 / 22)$ had type A while of those presumably infected by sharing injecting drug equipment $75 \%(21 / 28)$ had type B. One possible explanation is that African strains of the virus are transmitted heterosexually more easily than typical strains from North America and Europe. This work has implications for HIV surveillance. For example, it would be extremely useful to know what proportion of HIV infections found in the ante-natal unlinked anonymous testing programme appear to have originated from Africa.

Lastly on transmission, Shearer from the US National Cancer Institute presented data showing that $\mathrm{T}$-cells from some high-risk anti-HIV negative, PCR-negative individuals appear to recognise synthetic HIV peptides, suggesting that they have encountered the virus (WeA1047). The proportion of highrisk people with positive responses was high, in while that for controls with no known risk was low. Should these results be confirmed, one of the potential implications might be that the test could be used as a surveillance tool, giving an early warning of increased levels of HIV exposure.

Progression of HIV and potential cofactors

The average time for individuals to progress to AIDS after seroconversion was again estimated in several groups. These results are $\overleftarrow{c}$ given in table 1 . The most striking finding is $\widetilde{\Phi}$ the extremely rapid rate of progression to $\overrightarrow{0}$ AIDS reported in Nairobi commercial sex $\omega$ workers (CSW) (PoC4353). To my knowledge this is the only study to have directly estimated the median time to AIDS in any African country, so interpretation is difficult. However, another study from Senegal followed 24 women with HIV infection for almost 3 years during which time only 3 developed AIDS (WeC1066). The high incidence of other STDs in the Nairobi CSWs could explain their poor clinical course. Two studies (PoC4325, PoC4400) found the presence of other STDs to be associated with more rapid progression to AIDS. In contrast, however, one report from the San Francisco City Clinic cohort found that HSV infection was associated with slower progression to AIDS (PoC4398).

The lower progression rates to AIDS in ơ IDUs and haemophiliacs compared with $N$ homosexuals (table 1) could be explained by the near-complete absence of Kaposi's sarcoma and the younger average age in the 0 former groups-the association between age and progression to AIDS was confirmed by ? many studies (PoC4398, PoC4410,

Table 1 Studies reporting rate of progression from seroconversion to AIDS at the Amsterdam conference.

\begin{tabular}{lllllc}
\hline Abstract No. & Risk group & Location & No. in cohort & $\%$ progressing & at $X$ yrs \\
\hline PoC4347 & homosexuals & Amsterdam & 348 & $50 \%$ & $9 \mathrm{yrs}$ \\
PoC4463 & homosexuals & Vancouver & 127 & $30 \%$ & $7.5 \mathrm{yrs}$ \\
MoC0065 & homosexuals & Holland/USA & 373 & $50 \%$ & $10 \cdot 5 \mathrm{yrs}$ \\
ThC0572 & homosexuals & San Francisco & 562 & $69 \%$ & $12.5 \mathrm{yrs}$ \\
PoC4449 & haemophiliacs & Italy & 732 & $50 \%^{\star}$ & $15 \mathrm{yrs}$ \\
MoC0062 & haemophiliacs & UK & 111 & $45 \%$ & $12 \mathrm{yrs}$ \\
MoC0066 & IDUs & Edinburgh & 306 & $23 \%$ & $8 \mathrm{yrs}$ \\
PoC4474 & IDUs & USA & 136 & $29 \%$ & $6 \mathrm{yrs}$ \\
\hline
\end{tabular}


PoC4561, PoC0033, MoC0062, MoC0065, ThC1555).

Other factors for which some association with rate of progression of HIV was reported were cigarette smoking (PoC4407, WeC1030 but no association in PoC4383) co-infection with CMV (MoC0062 but no association in PoC4410), HTLVI/II (PoC4415,ThC1555), methadone use in IDUs (PoC4557), continued drug injecting in IDUs (PoC4561), lower socioeconomic status (PoC4742), race (more rapid $\mathrm{CD} 4$ decline in whites vs. blacks MoC0064). HLA type showed no consistent association with disease progression (MoC0087).

\section{Biological markers of progression}

The fundamental importance of the decline in CD4 lymphocyte number during HIV infection is now well accepted. Among other markers of progression, raised levels of non-specific immune activation markers (beta-2-microglobulin, neopterin, soluble interleukin-2, IgA and alpha-interferon) were again shown to strongly predict progression to AIDS (PoC4414, PoC4418, PoC4750, PoC4754, PoC4744, MoC0084, MoC0088). Two studies confirmed a previously published finding that those with a more severe primary acute seroconversion illness tend to progress more rapidly to AIDS than those for whom no illness was noticed (MoC0063, MoC0085).

There were some interesting data presented showing the association between viral load and risk of AIDS. A relative hazard of developing AIDS of 5.4 was found between individuals in whom virus was detectable using the polymerase chain reaction (PCR) and those in whom it was not detectable (MoC0083). Similar differences in CD4 count decline were observed. Andrieu and colleagues (TuA0559) measured cellular viral load by quantitative co-culture in 103 patients who were then followed for an average of almost 3 years. The results (table 2) indicate an extremely strong association with progression of HIV infection. Finally evidence was produced confirming that individuals harbouring syncytium-inducing HIV variants tend to lose CD4 cells and progress to clinical disease more rapidly than those with NSI HIV variants (PoA2061).

\section{Behavioural change-Graham Hart Introduction}

Each year the International AIDS Conference has a different and distinctive atmosphere and this year the emphasis was upon actionprevention programs and their evaluation.

Table 2 Progression of HIV infection according to cellular viral load (TuA0559)

\begin{tabular}{lllllll}
\hline $\begin{array}{l}\text { Frequency of } \\
\text { infected } \\
\text { cells }\end{array}$ & $\begin{array}{l}\text { No. of } \\
\text { subjects }\end{array}$ & $\begin{array}{l}\text { Initial CD4 } \\
\text { count }\end{array}$ & $\begin{array}{l}\text { Final CD4 } \\
\text { count }\end{array}$ & $\begin{array}{l}\text { Mean } \\
\text { months } \\
\text { follow-up }\end{array}$ & $\begin{array}{l}\text { Mean } \% \\
\text { CD4 count } \\
\text { decline } \\
\text { per year }\end{array}$ & AIDS \\
\hline$<1 / 3000$ & 83 & $737(339)$ & $584(334)$ & 35 & 7 & 5 \\
$>1 / 3000$ & 20 & $508(162)$ & $53(60)$ & 29 & 37 & 15 \\
\hline
\end{tabular}

Another new development was evidence of an interest in a wider range of population groups. I refer particularly here to the studies associated with mobility and potential for epidemic spread, notably as a result of international travel, sex tourism and cross-border labour or refugee movement. Another area with strong political interest and connotations entirely new to the Conference was the "problematising" of male heterosexual sexual activity. For example, one strand was entitled "changing male heterosexual attitudes and behaviour" whereas its female equivalent was "empowering heterosexual women", a very different strategy. That in the minds of some this is long overdue raises another set of issues entirely.

This presentation will continue previous years' practice of providing a report on new work in relation to gay men and drug users, and include interesting developments in relation to other groups, such as international travellers and sex workers.

\section{Gay Men}

The North Americans appear to be moving in two directions in relation to HIV prevention; one is to investigate and put resources into the prevention of so-called "relapse" which takes the individual as the focus of concern, the other is to use peer educators making formal and informal contacts with men known to them or contexts in which gay men meeta group or community based intervention. Two of these are worth describing in some detail, as they are relatively new in the AIDS field. The first is the Men's Network, a community-level approach for young (aged 18-28 years) gay men, in California. It is taking place in three medium-sized cities sequentially; data are currently available for the first. Baseline data were collected six months and immediately prior to the intervention, followed by questionnaires distributed immediately post-intervention, then six months and one year later.

The programme consisted of peer outreach, which included formal presentations in bars and at community events and informal contacts in popular cruising areas. The network itself is made up of men recruited from the community, is peer-run and the programme peer-designed; the network has a turn-over of members as new people are recruited and leave. Preliminary findings were able to compare men "highly exposed" (had participated in two or more events, were network members or had attended workshops) and those "not highly exposed". Although there was a population based increase in norms supporting safe sex, these were higher in the most exposed group, as was reported in discussion with friends and communication with sexual partners about safe sex, reported enjoyment of unprotected sex fell in the highly exposed as compared with the less exposed group. This research is clearly at an early stage of development, but the findings are promising. In particular, a major aim of the project is that it become self-perpetuating, 
through the constant turnover of members of the network and the range of contacts made by the men en masse and individually (PoD 5749).

The second intervention, and one of the most impressive for gay men, was recounted by Kelly et al (TuD0543). Sixteen small US cities were selected, each with a relatively discrete and identifiable gay community, usually with two well-used gay bars. Eight of the cities were randomly designated as intervention centres, and eight as comparators. Baseline sexual behaviour measures were taken of 4500 men attending the bars in each of the 16 cities. In the intervention cities researchers identified several gay men who were well-known, liked and respected, and these were given training over a two-month period in the transmission characteristics of HIV, safer sex behaviours, and conversational means of introducing topics such as sexual risk reduction. They subsequently made it clear to bar attenders they met, and others at private parties and elsewhere, that they fully endorsed and had personally committed themselves to the safe sex philosophy. All cities received educational materials, including posters, leaflets and videos, but the comparison cities were not exposed to the newly informed and enthusiastic peer educators. After six months of study repeat surveys were undertaken. In the cities receiving the experimental intervention, there were systematic reductions in population risk behaviour, with decreases from baseline measures of 20 $25 \%$ in the proportion of men reporting high risk sex, and corresponding reductions in the frequency of such behaviour. Little change occurred in the "comparison" (non-intervention) cities.

There are three major problems with the study. Firstly, the six month follow-up is insufficient to determine the efficacy of the intervention. Secondly, it may be that in the intervention cities the men who were the recipients of safe sex conversations were more likely in the self-reports on risk behaviour in the surveys to describe safe rather than unsafe sex if it were perceived that the latter was a highly stigmatised activity. Finally, perhaps the most persuasive verification of the intervention will come a year or so later, when measures of STDs and, most importantly the incidence of HIV infection, should be lower in the intervention cities than in those where only written and audiovisual materials were available.

Although in the West we often decry the value of printed materials in health education as an exclusive means of influencing behaviour change, in cultural contexts where there is high levels of literacy but virtually no information on HIV infection these may have a value and effect greater than that seen in cultures "overburdened" with information. This appears to have been borne out in Czechoslovakia, where leaflets funded and produced by the Dutch Ministry of Health were distributed in gay clubs and saunas which, though not specifically gay, attracted a significant number of men having sex with men. In December 199114000 of these leaflets were distributed, the most immediate consequence of which was a substantial increase in condoms sold, with the result that the Czech producers of condoms approached one of the country's few gay organisations ("Lambda") to discuss follow-up activities. Full evaluation is awaited, but it is interesting to note that sophisticated and costly interventions are not always the most effective on a mass scale (Hendriks et al, PoD5127).

\section{Drug users}

In a remarkably frank poster from New York, Ernest Drucker, the Director of a number of treatment programs, evaluated the national effectiveness of treatment-based interventions for drug users (PoC4250). More than $90 \%$ of such treatment in the USA is "high threshold", that is, employs waiting lists, contracts and other barriers to entry. However, less than $15 \%$ of the country's drug users are in treatment (methadone maintenance or therapeutic communities) at any one time and, in relation to methadone maintenance, more than $50 \%$ of IDUs in treatment continue to inject. It is worth quoting Drucker verbatim. "High threshold drug treatment reaches only a small proportion of IDUs and fails to retain most of them for sufficient time to effect AIDS risk behaviour. Low threshold treatment and harm reduction strategies (for example needle exchange) can reach many more IDUs at far lower costs, producing superior population outcomes". The Directors of few intervention programs would be willing to be so candid. This is not to say that for those who are engaged in methadone maintenance programs there is no reduction in risk behaviours, as was demonstrated in a number of posters (PoC4257; PoC4262; PoC4268; PoC4282). AIDS education interventions also reportedly had positive outcomes (PoC4277; PoC4279; PoC4794; PoD5091), as did individual counselling (PoC4800) although this was not a universal finding (PoC4788). Such AIDS education programs were associated with reduced incidence of both HIV and HBV in some settings (PoC4797).

Outreach efforts to drug users reportedly had positive outcomes in a number of posters (PoC4255; PoD 5075; PoD5085) and continued evidence supports the efficacy of needle-exchange and increased needle and syringe availability as safe and effective public health interventions (PoC4256; PoC4283; PoC4284; PoC4801; PoD5076); this is apart from their popularity with drug users (PoC4795).

Although it could be argued that needle-exchange and outreach programs are community-based interventions-the aim is to change social norms rather than offer individual counselling - there are fewer reports in the drugs field which highlight the role of community support for safer behaviours. One exception is to be found in Abdul-Quader et al (PoD5489) who found that safer sex 
behaviour in the non-injecting female partners of male injecting users was associated with peer influence and social norms, emphasising the potential that interventions such as those reported above in studies of gay men may also be effective in populations where drug use is highly prevalent. The possible value of such approaches was also reported by Faruque et al (PoD5491) when investigating the sexual risk behaviour of male crack users in Harlem.

\section{Other groups at risk}

Although there were a large number of presentations on prisons and prisoners (PoD5053-72), few of these reported on interventions and those that did were mostly concerned with improving knowledge and attitudes in relation to HIV/AIDS; many were awaiting full evaluation. Similarly, much of the work undertaken on female sex workers was descriptive (PoC4178-4196; PoD5600-5679), demonstrating (a) low levels of infection in prostitutes in Europe who do not also inject drugs, (b) high levels of HIV infection in sex workers in subSaharan Africa and (c) levels of condom use varying according to the degree of women's economic power and control, and competition from other sex workers. There were however some reports on interventions undertaken, including trial use of the female condom (PoC4180; PoD5647). One of the most innovative interventions for sex workers and their clients took place in Chiang Mai, Thailand (PoC4185). This involved small group training for prostitutes, selecting a key sex worker in each brothel to continue peer education, improving relations between brothel owners/managers and public health workers, and promotion of condom use by the use of signs such as "We welcome only guests using condoms". The outcome of this intervention in 500 customers was a reported increase from $10-20 \%$ condom use to $80-94 \%$ usage in different brothels. Economic savings to brothel owners were in the range $\$ 200-250$ per month as a result of a reduced incidence of STDs in sex workers.

Unusually there were also reports from the UK of risk behaviours in the male clients of prostitutes in the UK (PoD5602; PoD5611), from the USA (PoD5616), Thailand (PoD5671) and Germany (PoD5677) a focus that has long been missing from the literature in the area.

Related to the work undertaken on prostitutes, particularly in Thailand and the Philippines, there has been some recent research on sex tourism and, more generally, sexual risk in international travellers. For example Hawkes et al (PoD5225) reported that of heterosexual men from WHO Pattern I countries reporting new sexual contacts abroad, $25 \cdot 7 \%(19 / 74)$ had paid for sex, with $52 \%$ of these $(10 / 19)$ maintaining that they always used condoms. Levels of condom use were higher in gay male sex tourists to Thailand (PoD5239) and Bali (PoD5240) although some risk behaviour was evident.

\section{Virology-Malcolm Semple}

Data from Jaap Goudsmit's Group represented the consensus of opinion regarding the natural history of HIV-1 infection. Two to 4 weeks after infection viraemia, p24 antigenaemia and cellular HIV-1 mRNA peek and then fall within another 2 to 4 weeks as the individual seroconverts. Integrated proviral DNA also peeks transiently but slightly later than the other virological marker. Some groups described a homology of genotypes in V3 region of the env gene rescued from patients during and shortly after seroconversion, suggesting a genetic bottleneck during transmission (PoA2105). This could lead to more accurate targeting of vaccines to specific virus types. $\mathrm{HIV}-1$ viraemia as determined by molecular and culture techniques was consistently found to be lower during the asymptomatic stage of infection (CDC disease stages II and III) than during symptomatic disease stages (CDC disease stage IV) (TuA0557, PoA2113), perhaps with the exception of those patients who only had HIV-1 associated Kaposi's sarcoma whose viraemia lay somewhere between. The virus type during asymptomatic disease does not induce the formation of syncytia (multinucleated giant cells), in culture with MT-2 cells whereas during symptomatic disease the virus type would appear to induce syncytia in culture (PoA2061). These data were corroborated by gene mapping which demonstrated that virulence at different disease stages was primarily influenced by the $\mathrm{V} 3$ env region and to a lesser degree by the V2 and V5 region (MoA0073).

As more sensitive molecular techniques are employed to detect HIV-1 in vivo, the described levels of viraemia and proportion of infected cells has increased to the extent whereby a direct cytopathic effect of HIV-1 on infected cells is a plausible mechanism for CD4 cell depletion (TuA0557, PoA2124 and PoA2138). Such techniques may have use in measuring disease progression and drug efficacy given the paucity of clinical markers for therapeutic efficacy, especially in asymptomatic patients.

Much media attention was focused on reports of "a new AIDS virus". This occurred after a number of speakers described subclinical and clinical immune deficiency in patients who HIV-1 or HIV-2 infection could not be demonstrated by serology, tissue-culture or molecular techniques. Such reports were over interpreted by the popular press and their significance exaggerated. In all there were approximately 40 such cases reported anecdotally at the conference. Given that there are approximately 20 million cases of HIV infection world-wide, the clinical significance of these new cases at this time is small $(1: 1000000)$. Meanwhile the Center for Disease Control, Atlanta, USA (CDC) is to co-ordinate an international study of such patients in an attempt to identify if there is a third virus in responsible or if there is a less dramatic explanation. 


\section{Immunology}

An alteration of the function of TH1 and TH2 helper cells which was associated with disease progression. TH1 cells are the helper cells which mediate the cellular immune response while TH 2 cells are the helper cells which mediate the humoral (antibody) response. A group demonstrated by in vitro studies of helper cells from healthy HIV-1 infected subjects that TH1 helper cell function was dominant and characterised by the capability to produce interleukin-2 (IL-2). This function was also typical of healthy uninfected normal controls and for this reason he suggested that such cellular function was to some extent protective. As HIV-1 disease progressed, $\mathrm{TH} 1$ cell function decreased and TH2 cell function became dominant and characterised by the production of interleukin-4 (IL-4). The addition of antibodies raised against IL-4 to helper cells from moderately symptomatic subjects in vitro restored TH1 cell function. With severe symptomatic disease, greatly reduced levels of both $\mathrm{IL}-2$ and IL-4 were observed indication a functional deficit in both cell types (WeA1047).

An important question from the clinical researchers was what were the best methods for evaluating vaccine and anti-viral efficacy? And this question may now have been answered by the recent improvements in the methods for quantifying cell-free viral load using molecular techniques such as quantitative reverse transcribed PCR (TuA0557 and PoA2134). While caution must be exercised when employing such surrogate markers they may give an early indication of efficacy. Vaccine research to date has been slow. Development has been hampered by the great geographical variation of the viral envelope and the evolution of variation of the envelope within a host over time. However clinical trials of active post exposure vaccines will soon start.

\section{Resistance}

The molecular basis of drug resistance has been further investigated. It is now possible to accurately quantify point mutations in pol associated with drug resistance and point mutations in env associated with increased virulence (PoB3327). The technique for the quantification of such point mutations permits the monitoring of the evolution of resistance to antiretroviral therapy and may in future permit "personalisation" of therapy and aid the design of combination drug therapy which minimise the development of drug resistance.

The benefit of ddI mono-therapy in early disease was described although CD4 counts only rose for 10 to 20 weeks before returning to baseline values (Merigan $T$, commercial satellite meeting). Point mutations at codons 78 and 184 were described which conferred resistance to dideoxyinosine (ddI) and partial cross-resistance to zidovudine (Hammer SM, commercial satellite meeting).

All of the non-nucleoside reverse transcriptase inhibitors (NNRTI) described quickly selected resistant virus in culture and none were effective against HIV-2. Point mutations in the HIV-1 pol gene at codons 181 and 103 are rapidly selected and conferred resistance to some NNRTIs (Dolin $R$, commercial satellite meeting). This does not bode well for their clinical use as mono-therapy. Better results were demonstrated using NNRTIs and conventional nucleoside reverse-transcriptase inhibitors in combination therapy, in particular one novel drug $b$-HAPPe in combination with zidovudine would appear to totally inhibit HIV-1 replication in vitro.

In summary, the emphasis at the international conference is shifting further towards clinical trials and physician-led research. There was less pure virology being described and instead scientists were concentrating on developing a range of credible research tools for use in large drug trials.

\section{Opportunistic infections-Melinda Tenant-Flowers}

Mycobacterium avium Complex (MAC)

The results of the ACTG 157 trial of clarithromycin treatment for disseminated mycobacterium avium complex (MAC) were presented by Chaisson (WeB1052). Seventytwo patients with symptoms and blood cultures positive for MAC were enrolled, had blood cultures taken 2-weekly for 12 weeks and were randomised to receive 2000,1000 or $500 \mathrm{mg}$ bd of clarithromycin. There was a significant dose related decrease in MAC bacteraemia and symptoms were also alleviated. Resistance occurred in $22 \%$ of patients by week 12 and 16 patients experienced dose related gastrointestinal side-effects necessitating discontinuation of therapy. Cameron presented a study of rifabutin for MAC prophylaxis (WeB1055). Two hundred and seventy four patients were randomised to receive rifabutin and 282 received placebo, end points were MAC bacteraemia or toxicity, and patients were followed for six months. There was a significant decrease in MAC bacteraemia $10 \%$ versus $17 \%$ in the placebo group, and a significant increase in MAC-free survival in the rifabutin group. There was no difference between the two groups with regard to adverse events, survival overall or liver function tests. Other posters presented at the conference showed increased survival with treatment for disseminated MAC 6.5 months v 2 months, (PoB3352); that rifabutin is a safe drug (PoB3625) with less hepatotoxicity than rifampicin, which may delay or prevent MAC bacteraemia (PoB3081).

\section{Cerebral toxoplasmosis}

Early studies using hydroxynapthoquinone BW566C80 as salvage therapy for persons intolerant to sulphadiazine and pyrimethamine (PoB3185) and as induction therapy together with pyrimethamine (PoB3199) were presented and further information is awaited.

\section{Herpes simplex virus}

Kessler (WeB1056) presented data on the 
treatment of AIDS patients with acyclovir resistant herpes simplex virus with topical trifluridine. Twelve patients were given open label $1 \%$ ophthalmic ointment tds for $10-14$ days if the lesion healed or for up to six weeks. By six weeks six had healed, two had partially healed and four had failed, although two out of the latter group may have been non-compliant. There were two cases of virological resistance and no toxicity. Safrin (ThB1548) defined risk factors for the development of acyclovir resistant herpes simplex in a case control study and described an association with chronicity of the lesions and with CD4 counts $<100$ cells $/ \mathrm{mm}^{3}$

\section{Cytomegalovirus}

Several small studies using intravitreal ganciclovir for $\mathrm{CMV}$ retinitis were presented (PoB3151, PoB3157, PoB3251) which suggested reasonable response rates.

\section{Cryptosporidiosis}

There is still no specific treatment for this condition. A study by Raventos A (PoB3239) showed the median survival of 66 patients was ten months, which was longer than previous studies had indicated. Small phase I studies of roxithromycin (PoB3209), aminocitidine (PoB3229), and letrazuril (PoB3257) were presented. For symptomatic relief, opiates remain the mainstay of treatment and for disease prevention boiling water to $60^{\circ} \mathrm{C}$ kills the organisms. Microsporidiosis is due to two groups of organisms, either Enterocytozoon bieneusi or Encephalitozoon species and is not a AIDS defining illness. However, two studies (PoB3340, PoB3344) showed the prevalence to be 25 and $20 \%$ respectively in HIV positive patients with diarrhoea.

\section{Bacillary angiomatosis}

Koehler (PoB3339) isolated and cultured the causative organisms (Rochalimea $s p p$ ) from cutaneous lesions which resemble Kaposi's sarcoma but may be amenable to erythromycin treatment (PoB3217).

\section{MOPPS — multiple opportunistic pathogen prophylaxis strategy.}

The aim of MOPPS is to use the fewest agents to prevent the largest numbers of opportunistic infections, and the optimum timing to introduce prophylaxis would be determined by the CD4 count. Two posters were presented at the conference concerning this strategy. Bihari (PoB3144) studied 88 patients who received cotrimoxazole, dapsone, pyrimethamine, fluconazole, acyclovir, chlarithromycin and ciprofloxacin for 8.8 months. Seven opportunistic infections occurred in this group but none of these represented breakthroughs on prophylaxis. There were 38 side-effects, mainly skin rashes and no drug interactions. Weiser J (PoB3278) presented a phase I study on 23 HIV-positive patients, CD4 counts were below 200, who received antiretroviral therapy, acyclovir, fluconazole, ciprofloxacin and cotrimoxazole. After 4.3 months there were no withdrawals, compliance was good apart from acyclovir and there has been one opportunistic infection due to MAI. Further studies are needed to evaluate whether prevention of disease which may be necessary for many years, can be safely carried out without risk of resistance and whether it is cost effective.

\section{Opportunistic infections-Annemiek de Ruiter}

\section{Pneumocystis carinii pneumonia (PCP)}

There were a large number of poster presentations covering various aspects of the management of PCP. One study of 153 patients (PoB3284) found aerosolised pentamidine, cotrimoxazole and dapsone with pyrimethamine to be equally effective for primary PCP prophylaxis, whereas a smaller study of 106 patients (PoB3312) concluded that cotrimoxazole, although resulting in the higher number of side effects, offered greater protection when compared with aerosolised pentamidine. In this particular study the use of cotrimoxazole appeared to offer some additional protection against toxoplasmosis.

There were a number of posters examining the use of dapsone for PCP prophylaxis. Two studies, (PoB3315, PoB3321) with a total of 724 patients concluded that dapsone, with or without pyrimethamine was as effective as aerosolised pentamidine for PCP prophylaxis although it's use was associated with a greater number of side effects.

A French study (We1017) compared 300 mg of aerosolised pentamidine monthly with dapsone $50 \mathrm{mg}$ daily and pyrimethamine $\mathbf{5 0}$ mg weekly for primary prophylaxis against both PCP and cerebral toxoplasmosis in 363 patients with CD4 counts of less than $200 / \mathrm{mm}$. There were 10 episodes of PCP in each group but significantly more treatment limiting side effects in the dapsone/ pyrimethamine group. There were significantly fewer episodes of cerebral toxoplasmosis in the group receiving dapsone/ pyrimethamine when compared with those receiving pentamidine (19 of 32 ) and this study was terminated on the basis of the protective effect of dapsone/pyrimethamine with respect to cerebral toxoplasmosis.

Two oral presentations investigated the use of alternative treatment regimes for PCP. A randomised double blind study (WeB1019) compared the safety and efficacy of oral $566 \mathrm{C} 80$ with oral cotrimoxazole in 322 patients with PCP. In patients with mild PCP $(n=236) 63 \%$ of patients in both groups met the criteria for therapeutic success. Therapeutic failure due to treatment limiting drug toxicity occurred significantly more frequently in the cotrimoxazole group, but therapeutic failure due to inadequate response was more frequent in those receiving $566 \mathrm{C} 80$ resulting in similar overall efficacy.

A Canadian group presented the results of a randomised double-blind trial (We1020) comparing clindamycin and primaquine with cotrimoxazole for the treatment of first episode PCP in 65 patients. Although diar- 
rhoea and rash were more common in the clindamycin group, and nausea and hyponatraemia more frequent in the group receiving cotrimoxazole there was no significant difference regarding efficacy, tolerance, use of steroids, mortality and PCP-free interval between the two groups and this study has formed the basis of a larger multicentre trial.

A second group from Canada presented the results of a small randomised placebocontrolled trial (WeB1016) evaluating the effect of oral corticosteroids in 23 patients with mild first episode PCP. Patients with a baseline oxygen saturation of greater than or equal to $90 \%$ were randomised to receive either $60 \mathrm{mg}$ of prednisolone daily followed by a tapering dose over the next 2 weeks, or placebo. Early deterioration in oxygen saturation occurred in significantly fewer patients in the steroid group, and there was a significantly improved exercise tolerance in this group, when compared to the placebo group which persisted to day 30. Concerns were raised over the small number of patients studied and the possible adverse effects of the use of steroids in HIV positive patients.

Finally, regarding the treatment of PCP a study was presented from the USA (PoB3297) which evaluated the use of trimetrexate for patients who were intolerant to pentamidine and cotrimoxazole, intolerant to one and refractory to the other or refractory to both. A total of 575 patients were evaluable of whom $38 \%$ were ventilator-dependent on entry. Overall $40 \%$ of patients responded to treatment which was defined as being alive one month after receiving at least 14 days of trimetrexate, and it was concluded that trimetrexate was well tolerated and effective in patients with limited therapeutic options.

\section{Neurology-Brain Sweeney \\ Classification and nomenclature}

McArthur (AIDS conference session 150, no abstract) discussed the recent definitions of clinical HIV-associated neurological syndromes proposed by the American Academy of Neurology Task Force on AIDS (table 3) and Budka (NS pg 17) reviewed the classification of neuropathology agreed by an international working party of pathologists and neurologists (table 4). ${ }^{4}$

\section{Epidemiology}

McArthur and Sines (NS pg 115) presented data from the MACS study of gay and bisexual men. Four percent had dementia at the time of AIDS diagnosis, $7 \%$ with AIDS developed dementia annually, and the cumulative incidence of dementia in AIDS was $15 \%$. Predictive factors for developing dementia included anaemia before AIDS onset, older age at AIDS onset, low body mass at AIDS onset and constitutional symptoms before AIDS onset. There was no predictive value of initial $\mathrm{CD} 4$ count, lymphocyte count, beta 2 microglobulin level, neopterin level, rate of $\mathrm{CD} 4$ decline or zidovudine use prior to developing AIDS.
Table 3 Nomenclature and research case definitions for neurologic manifestations of human immunodeficiency virus-type-1 (HIV-1) infection ${ }^{2}$ (American Academy of Neurology 1991;41:778-785)

Central Nervous System Disorders:

HIV-1-associated dementia complex ${ }^{\star}$

HIV-1-associated myelopathy ${ }^{\star}$

HIV-1-associated minor cognitive/motor disorder

HIV-1-associated progressive encephalopathy of childhood ${ }^{\star}$ Peripheral Nervous System Disorders:

HIV-1-associated acute inflammatory polyradiculopathy

syndrome (Guillain-Barre syndrome) ${ }^{\star}$

HIV-1-associated predominantly sensory polyneuropathy ${ }^{\star}$ HIV-1-associated myopathy ${ }^{\star}$

${ }^{\star}$ All of these disorders can be classified as either probable or possible.

\section{Neuropathology}

Budka (NS pg 17) reported that $20-40 \%$ of AIDS patients have HIV encephalitis or HIV leukoencephalopathy at necropsy. One third of these have HIV encephalitis; one third HIV leukoencephalopathy; and one third a combination of these two processes. Budka's analysis of clinicopathological correlations suggested that the severity of HIV associated Dementia Complex correlated with the severity of some neuropathological changes, such as, astrocytosis, myelinic vacuoles. McArthur (AIDS conference session 150, no abstract) and Glass (NS pg 70) found that multinucleated giant cells and myelin pallor are relatively specific for dementia when present but that up to $50 \%$ of patients which clinical dementia do not have these abnormalities at necropsy.

\section{Pathogenesis}

Quinolinic acid and gp120 have been shown to be neurotoxins in vitro and act by stimulating the NMDA (N-methyl-D-asparate) glutamate receptor. Heyes and Brew (NS pgs 6, 9, $53,54,105)$ presented data suggesting that quinolinic acid, a metabolite of tryptophan, is an important neurotoxin in AIDS. Lipton (NS pg 5) gave the current evidence regarding gp120 and Giuillan (NS pg 7) presented data indicating that the neurotoxin was an asyet-unidentified low molecular weight protein also acting at the NMDA receptor. Agents which might block this NMDA receptormediated neurotoxicity are still some way from being used in clinical trials. While all authors stress the importance of glial cell, macrophages and $T$ cells in the aetiology of brain dysfunction, Giulian, Pulliam, Gendelman, Glass, Jassoy (NS pgs 7, 8, 51, 70,49 ) in particular presented their work on cellular mechanisms of neurotoxicity.

\section{Imaging}

Hall-Craggs et al (NS pg 73) and Ainsworth (PoB 3759) presented data on proton magnetic resonance spectroscopy of cerebral white matter in patients with HIV infection and controls showing relative reductions in the putative neuronal marker $\mathrm{N}$-acetyl aspartate (NAA) in patients with AIDS compared with controls and in AIDS patients with neurological deficits compared with those with no deficits. 
Table 4 Recommended neuropathology-based terminology of HIV-associated disease of the nervous system (Budka et al. Brain Pathology 1991;1:143-152)

\begin{tabular}{lll}
\hline 1. & Central Nervous System Disorders: \\
$1 \cdot 1$ & HIV encephalitis \\
$1 \cdot 2$ & HIV leukoencephalopathy \\
$1 \cdot 3$ & Vacuolar myelopathy and vacuolar leukoencephalopathy \\
$1 \cdot 4$ & Lymphocytic meningitis \\
& $1 \cdot 5$ & Diffuse poliodystrophy \\
2. & Peripheral Nervous System: \\
$2 \cdot 1$ & HIV-1-associated acute inflammatory demyelinating polyradiculoneuropathy \\
$2 \cdot 2$ & Chronic inflammatory demyelinating polyradiculoneuropathy \\
$2 \cdot 3$ & HIV-1-associated predominantly sensory axonal neuropathy \\
$2 \cdot 4$ & Ganglionitis, ganglioradiculitis, polyradiculoneuritis \\
& $2 \cdot 5$ & Necrotising vasculitis, vasculitic neuropathy \\
Skeletal Muscle: \\
$3 \cdot 1$ & Polymyositis \\
3.2 & Necrotising myopathy \\
3.3 & Nemaline rod myopathy \\
3.5 & Necrotising vasculitis \\
\hline
\end{tabular}

\section{Neuropathy}

Schaumburg (NS pg 13) discussed the peripheral neuropathies caused by the nucleosides $\mathrm{ddC}$ and $\mathrm{d} 4 \mathrm{t}$. The neuropathy caused by ddC is related to the dose of treatment given: high doses result in a rapid onset, crescendo distal neuropathy; low doses cause a gradual onset, less intense sensory neuropathy. Both respond to withdrawal of the drug. The role of nerve growth factor in treating the distal sensory neuropathy of HIV infection has not been defined.

\section{Progressive multifocal leukoencephalopathy (PML)}

Berger (NS pg 14) presented data from a series of 70 patients with PML. Ten percent of cases have a protracted course without specific intervention. Cytosine arabinoside, adenine arabinoside, idoxuridine, zidovudine, alpha interferon, beta interferon and corticosteroids have all been used to treat PML but none is clearly beneficial. Whiteman (NS pg 38) retrospectively analysed $C T$ and MRI scans of 49 patients with pathologically proven PML. Lesions were typically parietooccipital or frontal in location and the majority involved periventricular and subcortical white matter. Lesions were also noted in the posterior fossa, corpus collosum, thalamus and basal ganglia. Contrast enhancement occurred in four patients and MRI was more sensitive to extent and number of lesions.

\section{Primary cerebral lymphoma (PCNSL)}

Levy (NS pg 46) presented a prospective study of radiotherapy in 15 patients with biopsy-proven disease. Ten patients completed a course of 3000 to $4000 \mathrm{cGy}$ given over 3 weeks in 15 fractions. The study found that radiotherapy does not have a major impact on CNS morbidity and mortality in PCNSL.

\section{Cytomegalovirus (CMV) polyradiculopathy}

Brew (NS pg 85) reviewed four patients who responded to ganciclovir and found that the features of lumbar pain, urinary incontinence, abnormal roots on myelogram and CSF polymorphonuclear pleocytosis were not present in all cases. $\mathrm{He}$ suggests that CMV polyradiculopathy is a diagnosis of exclusion and that patients with negative tests may require a therapeutic trail of ganciclovir or foscarnet. Assuerus (NS pg 44) identified two patterns of CMV neuropathy in a group of 14 patients: a polyradiculopathy affecting the lower limbs and a "mononeuritis multiplex" which can involve the upper limbs. Of 11 treated patients ten responded to ganciclovir or foscarnet initially but CMV encephalitis was the cause of death in six.

\section{Disease progression and survival- Danielle Mercey}

Most of the studies presented comparing survival between men and women showed no significant gender differences. The role of access to health care in the USA in determining outcome was much discussed. Sacks et al (MoC0030) in a retrospective comparison of 149 and 136 men matched for age, race and mode of acquisition of HIV found no significant differences in duration of follow-up or lag time from diagnosis to first follow-up visit. More women were asymptomatic at presentation, and they had significantly higher T4 cell counts, probably because many had been diagnosed at routine ante-natal screening. When matched for CDC stage at entry no significant differences in progression rates or survival were seen. Sherer et al (MoC0031) similarly showed, in a comparison of 700 women and 2963 men that women have higher T4 cell counts at enrolment. Apparent differences in rates of PCP prophylaxis and zidovudine administration were not real if adjustment was made for T4 cell count. No significant differences in progression or survival were seen. Creagh et al (MoC0032) in a study of 252 and 2606 men apparently showed a poorer survival for women with base line T4 cell counts of less than 100 , compared with men with similar counts, but acknowledged that this was probably a race related difference with white women and white men having similar survivals. American and African black women were less likely to receive zidovudine. The Italian Seroconverters Study (MoC0033) showed no significant difference in rate of progression of clinical disease, nor of T4 cell count decline between 257 women and 685 men. Similarly in the United Kingdom Brettle et al (MoC0066) showed no significant gender differences in clinical progression or survival in a cohort of 306 patients of known seroconversion time intervals.

\section{Clinical manifestations}

Five studies (0034) looked specifically for gender differences in AIDS defining diagnoses. Four of these studies found increased rates of oesophageal candidiasis amongst women but not all of these differences were statistically significant. Several studies commented on the low rates or absence of Kaposi's sarcoma in women. Wasting syndrome was diagnosed significantly more frequently amongst women than men in one study. (MoC0032). Many of these studies were criticised for using the CDC case definition for AIDS and not looking specifically for gynaecological conditions. However, since 
the CDC case definitions gave similar progression rates and survival times it seems unlikely that gynaecological conditions should also be regarded as AIDS defining.

Benson et al (MoC0034) noted that 32\% of their cohort of women had recurrent vulvo vaginal candidiasis (criteria for diagnosis were not defined). Eight percent had pelvic inflammatory disease and $10 \%$ cervical intra epithelial neoplasia. However, with no control group of women it is not possible to comment on the significance of these findings. Anastos et al (TuB0532) performed pelvic examinations and microbiological screening on 131 gynaecologically asymptomatic HIV positive women and found $19 \%$ to have vaginal candidiasis, $13 \%$ trichomonas vaginalis, $13 \%$ bacterial vaginosis, $7 \%$ pelvic inflammatory disease and $26 \%$ were normal. However, no association between the prevalence of these disorders and the degree of immune suppression as measured by $\mathrm{T} 4$ count was found.

\section{Cervical intra epithelial neoplasia (CIN)}

Many studies were presented exploring the relationship between $\mathrm{CIN}$, HIV infection and immune suppression. Klein et al (TuB0527) in a prospective, controlled study of $93 \mathrm{HIV}$ positive and $110 \mathrm{HIV}$ negative women on a methadone maintenance programme found significantly more human papilloma virus and CIN in HIV positive women and further found a correlation with lower T4 cell counts. They noted a good correlation between histology and cytology for their cohort but did not colposcope women with negative cytology. After a median follow-up of 17 months $33 \%$ of the HIV positive women treated for CIN had persistent disease but no recurrence was found. No invasive disease was detected. Anastos et al (TuB0532) in their study of 150 HIV positive women also showed a convincing correlation between cytological abnormality and T4 cell count or clinical disease stage.

Fruchter et al (MoB0057) compared 47 HIV positive and $157 \mathrm{HIV}$ negative women attending a colposcopy service for the presence of CIN1 or more severe abnormality on cytology. The stage of clinical disease for the positive women was not specified. They found more high grade lesions on the cervical biopsy, larger lesions and more vulvo vaginal lesions in HIV positive women. They also commented that they had a less good correlation between cytology and histology for HIV positive women and that this was probably due to a large proportion of the HIV positive cervical cytology specimens being obscured by inflammatory exudate. Treatment outcome was reported as worse for $\mathrm{HIV}$ positive women with $50 \%$ biopsy proven recurrence rates at one year. Recurrence was independent of the severity of the initial CIN or of the treatment modality used for HIV positive women. They suggest a need for colposcopic screening of patients and different treatment protocols.

Issues for women and children-Di Gibb Paediatrics

There were more data presented about paedi- atric aspects of HIV infection than at previous conferences, in particular on epidemiological aspects.

\section{Vertical Transmission}

Vertical. Transmission Rates (see table 5) Standardisation of methodology for calculating vertical transmission rates was discussed at a conference held in Ghent, Belgium in early 1992. This may have contributed to the fact that at this conference, there was less variation between vertical transmission rates reported from the different centres. Where problems such as loss to follow-up or death occurred prior to knowing the HIV infection status of the child (a problem particularly in Africa), some studies presented a possible range of transmission rates (eg PoC4226, WeC1058). In general the transmission rates reported from Africa are lower than previously reported in Europe agreed with the European Collaborative study (ThC1520) (see table 5). Data from CDC, Atlanta (WeC1057) showed that there was no increased risk of transmission to subsequent siblings born to an HIV-1 positive mother.

$H I V-2$ Preliminary data about vertical transmission of HIV-2 were presented from Cote d'Ivoire (WeC-1065). Seroprevalence among 12,750 antenatal women was $\mathbf{9} \cdot 5 \%$ for HIV$1,1.7 \%$ for HIV-2; $1 \%$ of women were infected with both viruses. Preliminary transmission rate data showed that $7 / 25(28 \%)$ of infants born to mothers with HIV-1 infection, and $1 / 34(3 \%)$ of infants born to HIV-2 infected women were infected. The diagnosis of infection was based on HIV IgA and PCR at 6 months of age. Birth weights of infants born to either HIV-1 or HIV-2 infected women were lower than in those born to uninfected women.

Early diagnosis and timing of vertical transmission Given the expertise to perform the investigations, HIV infection can now be diagnosed by 2-3 months of age in the majority of infants born to HIV positive mothers. However, there are a proportion of babies who are negative for HIV by all techniques during the first week of life, but positive by 1-3 months of age. It is increasingly felt that this indicates intrapartum acquisition of infection rather than lack of sensitivity of techniques for measuring HIV infection in

Table 5 Vertical transmission rates 1992

\begin{tabular}{lrll}
\hline & Number & Rate & Abstract \\
\hline Europe & & & \\
ECS & 721 & $14 \cdot 4 \%$ & ThC1520 \\
Barcelona & 125 & $16 \%$ & PoC4235 \\
$\begin{array}{l}\text { Madrid } \\
\text { Switzerland }\end{array}$ & 167 & $18 \cdot 5 \%$ & PoC4229 \\
USA & 31 & $12 \cdot 9 \%$ & PoC4223 \\
$\begin{array}{l}\text { New York } \\
\text { Collaborative study }\end{array}$ & 250 & $28 \%$ & WeC1059 \\
$\begin{array}{l}\text { Connecticut } \\
\text { Africa }\end{array}$ & 128 & $21 \%$ & PoC4215 \\
$\begin{array}{l}\text { Rwanda } \\
\text { Uganda }\end{array}$ & 185 & $21-36 \%$ & PoC4226 \\
Zaire & 411 & $16-30 \%$ & WeC1058 \\
Puerto Rico & 89 & $23 \cdot 1 \%$ & PoC4235 \\
& 202 & $26 \%$ & PoC4220 \\
& \multicolumn{3}{l}{ (at 6 months) } \\
\hline
\end{tabular}


blood. However, an alternative explanation could be that the virus is "hiding" in tissues in the first days of life and therefore not available for detection in blood. There were several presentations on methods for early diagnosis. These included viral culture, PCR (RNA and DNA), HIV specific IgA, and P24 antigen detection following acid dissociation in immune complexes. In general, viral culture and PCR were positive in only $30-50 \%$ of infected children at birth but this rose to $92-100 \%$ between by $1-3$ months of age (ThC1581, TuB0511, WeC1060, ThC1580). P24 antigen was much less sensitive $(16-18 \%)$ positive in the first week of life, rising to $25 \%$ by $1-3$ months (TuB0511, ThC1579). Sensitivity was increased if samples were acid treated to dissociate antigenantibody complexes, resulting in $80-90 \%$ sensitivity by 1-3 months of age (TuB-0512, ThC1579). HIV specific IgA was less useful with $30-70 \%$ sensitivity at $1-3$ months (ThC1580). In general false positives may occur in cord blood but specificity was reported to be very high thereafter. Caution must be exercised in attempting to estimate the proportion of infection occurring at the time of birth from these sensitivity data because the timing of testing varied in the different studies. However, assuming that virus is not 'hiding' in tissues in the early weeks of life, it is possible that between $50-70 \%$ of transmission occurs at the time of birth.

Evidence for intrauterine infection was discussed by Ayre Rubinstein in his State of the Art lecture-namely, the presence of HIV in early and mid-trimester foetuses (also described in PoA2466), early onset of PCP, low CD4 count and CNS symptoms in some infants, and the fact that viral or PCR techniques identify some infected babies in the first week of life (see above).

Further evidence for intrapartum infection included results from an update of the International Twins Study (WeC-1062), previously reported in Lancet 1991. Seventy (29\%) of 242 twins (121 sets) were infected. Of these, 38 sets of twins were discordant for HIV infection. In 31 sets $(26 \%)$ Twin A was infected, and in only 7 sets $(6 \%)$ was twin B infected. Of the twins followed prospectively, transmission rate was $23 \%$ in twin $\mathrm{A}$ and $6 \%$ in twin B. There was a trend for concordance of infection status with monozygotic twins, and progression of disease was similar where both twins were infected. In addition, in this study, Caesarean section (LSCS) was observed to be significantly associated with decreased transmission (22\% versus $35 \%$, p $<0.04)$. Although a tendency for lower transmission rate with LSCS has been observed in other prospective studies (such as European Collaborative study, ECS), and although the potential for bias exists in this study, it is none-the-less, the first to report a statistically significant difference.

Risk factors for transmission There were many presentations on the risk factors associated with vertical transmission of HIV infection. The largest study (ECS) has already published data ${ }^{4}$ and summarised findings in presentation ( $\mathrm{ThC}$ 1520). Increased transmission was associated with maternal CD4 count < $700 / \mathrm{mm}^{3}$, P24 antigenaemia, breast feeding (see below) and prematurity. Findings from other presentations are shown in table 6.

Breast feeding The European Collaborative study reported a doubling in the risk of transmission with breast feeding (Odds ratio $2 \cdot 25$ (95\% CI 0.97-5.23) (ThC1520). In this presentation data from a meta-analysis by Dunn et al, which has since been published in the Lancet $^{5}$ were also presented. The additional risk of breast feeding in women already infected during pregnancy was estimated from 6 prospective studies to be $14 \%$ (95\% CI 7-22\%). This risk of transmission from breast feeding where the mother acquired HIV infection postnatally was calculated from 4 studies to be $29 \%$ (16-42\%).

In an attempt to define which breast feeding mother transmitted to their offspring, studies from Rwanda (ThC1523) and Haiti (ThC1524) studied PCR in breast milk. PCR positivity was highest in colostrum, and in both studies was associated with higher vertical transmission rates from mother to child. However, in the Rwandan study, transmission was also independently associated with reported multiple sexual partners during pregnancy which might be a surrogate for primary infection. It is unclear whether the presence of PCR in breast milk is merely a marker for infectiousness of the mother or an independent factor contributing to vertical transmission via breast milk.

Table 6 Risk factors for vertical transmission

\begin{tabular}{|c|c|c|c|c|c|}
\hline \multicolumn{2}{|l|}{ Associated } & \multicolumn{2}{|l|}{ Preliminary Results } & \multicolumn{2}{|c|}{ Not Associated } \\
\hline $\begin{array}{l}\mathrm{P} 24 \mathrm{ag} \\
\text { ThC1520) }\end{array}$ & (ThC1520, PoC4216, & vaginal delivery & (WeC1062) & age & (ThC1524, \\
\hline $\begin{array}{l}\text { low CD4 } \\
\text { ThC1520) }\end{array}$ & $\begin{array}{l}\text { PoC4231) } \\
\text { (PoC4226, PoC4231, }\end{array}$ & lack $1 \mathrm{gM}$ in milk & $(\mathrm{ThC1521})$ & parity & (ThC1524, \\
\hline breast feeding & $\begin{array}{l}\text { WeC1059, ThC1520) } \\
\text { (ThC1520) }\end{array}$ & $\begin{array}{l}\text { multiple partners in } \\
\text { pregnancy }\end{array}$ & $(\mathrm{ThC} 1524)$ & race & $(\mathrm{ThC1524})$ \\
\hline $\begin{array}{l}\text { PCR in breast milk } \\
\text { ThC1524, }\end{array}$ & (ThC1524, ThC1521, & maternal viraemia & (PoC4228) & IVDU & (PoC4215, \\
\hline prematurity & $\begin{array}{l}\text { ThC1523) } \\
\text { (PoC4235, ThC1520) }\end{array}$ & $\begin{array}{l}\text { primary maternal } \\
\text { infection } \\
\text { lack of neutralising } \\
\text { antibodies }\end{array}$ & $\begin{array}{l}\text { (PoC4215) } \\
(\text { WeC1061) }\end{array}$ & & ThC1520) \\
\hline
\end{tabular}


It should be stressed that although breast feeding is not advised in situations where safe alternatives exist, WHO continues to recommend that in countries and in situations where safe alternatives to breast feeding do not exist, women with HIV infection should continue to breast feed their infants.

Natural history and clinical aspects There were little new data from prospective studies on the natural history of HIV infection in vertically infected children. Cross-sectional paediatric AIDS data from National Surveillance in Europe (PoC4242) reported that of 832 cases, $27 \%$ had developed AIDS in the first year of life. This is in agreement with prospective studies such as the ECS. However, they also estimated that $40 \%$ of children would develop AIDS by 2 years, and $52 \%$ by 3 years. These are likely to be overestimates of progression and further data from prospective cohorts are required to tell us the progression rates to AIDS in the $75 \%$ of infected children who do not develop AIDS in the first year of life.

A presentation by Anita de Rossi from Padua, Italy, of 17 children described an association between the presence of syncitial inducing, rapidly growing virus and a quantitative PCR of more than 500 copies $/ 10^{5}$ PBMC and the development of AIDS in the first 12 months of life (WeC1060). Further work is required to confirm whether such techniques will be useful in predicting which children might benefit from very early therapy. It was commented that treatment with antiretrovirals during pregnancy might affect both the ability to make an early diagnosis and to predict early progression of disease.

Three studies described early stunting in children with vertically acquired HIV infection (PoB3630, PoB3643). Weight for height centiles were near normal and the possibility of a neuro-endocrine cause requires further investigation.

There were few new data on therapy in children with HIV infection. Two studies on PCP prophylaxis suggest that early initiation of primary PCP prophylaxis based was successful in reducing the incidence of PCP in USA (PoB3641, PoB3642, PoB3664) but HIV needs to be diagnosed early enough to institute therapy.

Paediatric HIV infection in the British Isles Two posters described aspects of the active paediatric surveillance of HIV infection in the British Isles coordinated at the Institute of Child Health (ICH), London. Poster PoC4219 described the confidential register of children born to HIV positive mothers. Paediatric reports notified via the British Paediatric Surveillance Unit (BPSU), are linked with reports of pregnant women with HIV infection and with laboratory reports. Quarterly tables of these data are produced by the ICH and published in the Communicable Disease Report. By looking at the numbers of reports by district in the London area, and comparing these to num- bers obtained from unlinked anonymous HIV testing of Guthrie cards, it was possible to ascertain that only $22 \%$ of children born to positive mothers in London are known about.

Poster PoB3639 described the experience of PCP in children with HIV infection in the British Isles. Of the 140 reported children with definitive HIV infection by April 1992 19 had developed PCP. In the majority of children PCP developed at 3-4 months of age, causing $14 / 25(56 \%)$ of the paediatric AIDS cases in the first year of life among children born in the British Isles. Only $8 / 19$ $(42 \%)$ were known about prior to the diagnosis of PCP and the mortality from PCP was $58 \%$. Furthermore children who survived PCP went on to develop further AIDS indicator diseases, particularly CNS disease and had a poor prognosis.

\section{Antiretroviral treatment-Ian Williams 1. Early zidovudine}

Cooper et al (PoB3718) reported the results of a large randomised placebo controlled trial of zidovudine ( $500 \mathrm{mgs}$ bd) in 993 patients with CD4 counts $>400 \times 10^{6} / 1$ with a median duration of follow-up of 94 weeks. Haematological toxicity was low, 3\% with zidovudine and $2 \%$ on placebo $(\mathrm{Hb}<8 \mathrm{~g} / \mathrm{dl}$ and/or neutrophils $\left.<0.75 \times 10^{9} / 1\right)$. Although using a $1 \mathrm{~g}$ daily dose this rate is similar to the reported toxicity on $500 \mathrm{mgs}$ daily in ACTG 019.

Protocoled clinical disease progression (AIDS, severe ARC, group IV C2) was reduced with zidovudine in comparison with placebo $[\mathrm{n}=11 \quad(2 \%), \mathrm{n}=22 \quad(5 \%), \mathrm{P}$ $0 \cdot 0486]$. There was also a significant difference in the number of patients with a $\mathrm{CD} 4$ count declining to $<350 \times 10^{9} / 1,70(15 \%)$ on zidovudine, $130(25 \%)$ on placebo $(\mathrm{p}=$ $0.006)$. Overall after two years using both these protocoled endpoints there was a significant benefit in receiving zidovudine in patients with baseline CD4 cell counts $<750$, particularly $400-499 \times 10^{6} / 1$.

The high withdrawal rate and the "soft" protocol and non-protocol endpoints for the trial are criticisms but the study does provide support for those clinicians wishing to use zidovudine in asymptomatic patients to delay progression.

Follow-up data from the Veterans Administration co-operative study of early versus late zidovudine therapy in patients with CD4 counts between 200-500 $\times 10^{6} / 1$ (PoB3723), confirmed their recent reported findings of a reduced rate of progression to AIDS with early zidovudine use but no overall survival benefit.

\section{Monotherapy: $d d I$}

No study reported a head-on comparison between zidovudine with either ddI or ddC in patients who had never received anti-retroviral therapy. Several though reported on the use of ddI in patients previously treated with and tolerant to zidovudine. In one of these (MoB0079, ACTG 116B/117) persons who 
had been treated for at least 4 months with zidovudine were randomised to receive either ddI $750 \mathrm{mgs}$, ddI $500 \mathrm{mgs}$ or continue on zidovudine $600 \mathrm{mgs}$ daily. Overall, there were a significantly fewer deaths and new AIDS defining events in patients taking ddI at $\mathbf{5 0 0}$ mgs than those continuing on zidovudine. A similar trend was seen with ddI 750 mgs but did not reach significance. The clinical benefit was primarily seen in patients with ARC or asymptomatic $\mathrm{HIV}$ infection treated with either ddI 500 or 750 mgs. No significant difference was seen in patients with a previous AIDS diagnosis between the three treatment regimens but the number of persons included may have been insufficient to identify a benefit. In these persons treated with ddI CD4 counts increased transiently, while in those who remained on zidovudine counts continued to decline.

Montaner et al (MoB081) reported similar changes in CD4 count in patients changed to ddI after at least six months therapy with zidovudine. The study is on-going to assess the effect on clinical outcome.

The incidence of pancreatitis in persons receiving ddI was reported variously between $2.5 \%$ and $10 \%$ dependent on dose and time on therapy (MoB0079, PoB3003), with no significant difference between the rates in persons treated with ddI $500 \mathrm{mg}$ or $\mathrm{ZDV}$ in ACTG 116B/117 (MoB0079). Similarly there was no difference in the incidence of peripheral neuropathy $(14 \%)$ in the three treatment regimens in this trial.

Yarchoan et al (MoB0054) compared an alternating regimen of zidovudine $600 \mathrm{mgs}$ daily with ddI $500 \mathrm{mgs}$ daily in three week cycles with simultaneous treatment of zidovudine $300 \mathrm{mgs}$ daily and ddI $250 \mathrm{mgs}$ daily, in 41 patients with symptomatic disease and less than three months previous nucleoside analogue therapy. The simultaneous regimen resulted in a more enhanced and persistent rise in $\mathrm{CD} 4$ count than on the alternating regimen. CD4 counts returned to baseline by 26 weeks on the alternating regimen but remained well above baseline for up to 44 weeks on the simultaneous regimen.

Similar patterns of changes in CD4 counts were reported by Collier et al at a recent report session in a comparative study ( $n=$ 69) of multi-dose combination of ddI with zidovudine in patients with a CD4 count $<400 \times 10^{6} / 1$ and who had previously received $<120$ days of treatment with zidovudine. The enhanced effect on CD4 count was particularly noted in zidovudine naive patients and appeared to be independent of dose. The number of patients though in each group was too small to exclude a dose response.

If one accepts that an increase in CD4 count is a good surrogate marker of probable clinical benefit then these results are encouraging for the outcome of a larger phase II/III combination trials.

Whether acyclovir in combination with zidovudine is clinically beneficial remains uncertain. Youle et al (MoB56) reported a significant increase of probability of survival at one year of patients with symptomatic disease (CD4 count <150), previously treated with zidovudine when taking acyclovir 800 mgs qds compared to placebo $(0.23$ versus $0.39, p=0.018$ ). The rate of decline of CD4 count was similar in both groups and there was a high withdrawal rate from the trial. There was no difference between acyclovir and placebo in the development of CMV disease.

Preliminary analysis of the trial of zidovudine $600 \mathrm{mgs}$ daily in combination with or without $4.8 \mathrm{~g}$ daily of acyclovir in early symptomatic disease (PoB3585) showed a non-significant trend to an enhanced CD4 response in patients receiving the combination regimen.

\section{Surrogate markers}

Several studies investigated the correlation between change in surrogate markers and clinical outcome in trials of therapy. Graham et al (PoB3473) reported that the baseline or change in CD4 count were the best predictors of progression to AIDS in persons treated with zidovudine. The relative risk of progression to AIDS was reduced to 0.24 at 7-12 months from start of treatment in those persons whose CD4 count increased by 100 cells from baseline. Similar findings were reported in a further study of zidovudine (PoB3471) and of ddI (PoB3482).

These are retrospective analyses and the current or change in CD4 count may not predict the overall clinical response to treatment. Other markers particularly viraemia may be more useful, for example, patients positive for serum P24 antigen level had a higher rate of decline of CD4 percentage per year than those P24 antigen negative when treated with zidovudine (PoB3479). New measures of plasma viraemia may be more useful in correlating response to treatment of clinical outcome and resistance (PoB3500, PoB3602, TuA557, TuA555).

\section{New antiretrovirals}

Of the new nucleoside analogues several phase I studies were presented on stavudine (D4T), 3TC, and fluorothymidine (FLT). Surrogate marker data including an increase in CD4 count and suppression of P24 antigen were reported in all three, particularly D4T (WEB 1010, 1011, POB 3011, 3023). Neutropenia and neuropathy and raised transaminases were observed in $10-15 \%$ of 64 patients (WB1011) treated with D4T for between 3 months and 2 years. In contrast no serious side effects were reported in a dose escalation study of 90 patients treated between 15-48 weeks with 3TC. Changes in surrogate marker data were less impressive with minimal transient rise in $\mathrm{CD} 4$ count suggesting that higher doses may need to be used.

The main concern with non-nucleoside analogue reverse transcriptase inhibitors is the rapid onset of in vitro resistance within several weeks of starting therapy, which is reflected in the rapid rise in P24 antigen to pre-treat- 
ment levels following the initial decline (WEB 1013, MOB 0053). It is unlikely these will be used as monotherapy but they may find a role in combination.

\section{Therapeutic vaccines}

The use of recombinant GP160 to stimulate an enhanced immune response in patients with asymptomatic HIV infection was presented by several investigators (TUB 560, 561,562 and 563). In those persons that responded to the vaccine, there were enhanced or new humoral responses to several epitopes, increased lymphoproliferative responses and in two studies an increase in CD4 cell count over time. Further controlled studies will need to address whether the responders are self selected, the humoral responses are neutralising to various HIV strains and whether cellular immune responses can be activated.

\section{Kaposi's sarcoma}

Single agent treatment of Kaposi's sarcoma with liposomal doxorubicin and liposomal daunorubicin was reported in several studies of a small number of patients with advanced Kaposi's sarcoma, many of whom had previously failed to respond to standard chemotherapy (PoB3018, 3016, 3123, 3119). Partial response rates of between $60-100 \%$ within a few weeks of starting treatment were reported. Neutropenia appeared more common with liposomal doxorubicin.

1 Lazzarin A, Saracco A, Musicco M, Nicolosi A. Man to woman sexual transmission of the Human Immunodeficiency Virus. Risk factors related to sexual behaviour, man's infectiousness and women's susceptibility. Arch Int Med 1991;151:2411-6.

2 Report of a working party of the American Academy of Neurology AIDS Task Force. Nomenclature and research. Case definitions for neurologic manifestations of human immunodeficiency virus type 1 (HIV-1) infection. Neurology 1991;41:778-85.

3 Goedert Jj, Duliège A-M, Amos CI, Felton S, Biggar RJ, and the International Registry of HIV-exposed twins. and the International Registry of Hiv-exposed twins. 1991;338:1471-5.

4 European Collaborative Study. Risk factors for mother-tochild transmission of HIV-1. Lancet 1992;339:1007-12.
Dunn DT, Newell ML, Ades AE, Peckham CS. Risk of Dunn DT, Newell ML, Ades AE, Peckham CS. Risk of
human immunodeficiency virus type 1 transmission through breast feeding. Lancet 1992;340:585-8. 